\title{
ANÁLISE ECONÔMICA DO CONSÓRCIO SERINGUEIRA-CACAU PARA GERAÇÃO DE CERTIFICADOS DE EMISSÕES REDUZIDAS ${ }^{1}$
}

\author{
Michele Karina Cotta ${ }^{2}$, Laércio Antônio Gonçalves Jacovine ${ }^{3}$, Sebastião Renato Valverde ${ }^{3}$, Haroldo \\ Nogueira de Paiva ${ }^{3}$, Adonias de Castro Virgens Filho ${ }^{4}$ e Márcio Lopes da Silva ${ }^{3}$
}

\begin{abstract}
RESUMO - Este trabalho teve como objetivo analisar a viabilidade econômica do consórcio seringueira-cacau sem e com os créditos de carbono, considerando-se a possibilidade de essa atividade gerar Certificados de Emissões Reduzidas (CERs). A quantificação dos CERs e a análise econômica foram realizadas em um horizonte de planejamento de 34 anos, em que a implantação da seringueira ocorreu no primeiro ano e a do cacau, no quarto ano do projeto. A estimativa de carbono do consórcio foi obtida a partir de dados de biomassa arbórea. O estoque de carbono estimado no consórcio seringueira-cacau foi de 106,91 tC/ha, o que correspondeu a 393 CERs/ ha. Pelas análises realizadas, concluiu-se que: o estoque de carbono na biomassa arbórea, particularmente das árvores de seringueira, credencia o consórcio seringueira-cacau como uma atividade promissora na geração de CERs, tornando o consórcio uma opção de projeto do Mecanismo de Desenvolvimento Limpo, com potencial de aprovação pela Convenção Quadro das Nações Unidas sobre Mudanças Climáticas.
\end{abstract}

Palavras-chave: Hevea brasiliensis, Theobroma cacao, projeto de carbono e análise econômica.

\section{ECONOMIC ANALYSIS OF THE RUBBER-COCOA INTERCROPPING FOR GENERATION OF CERTIFIED EMISSION REDUCTION}

\begin{abstract}
The objective of the present work was to analyze the economic viability of rubber-cocoa intercropping with and without carbon credits, considering the possibility of this activity to generate Certified Emission Reductions (CERs). The CERs quantification and the economic analysis were accomplished for a 34-year planning horizon, in which the implantation of rubber tree occurred in the first year and cocoa in fourth year of project. The intercropping estimated carbon stock was obtained from arboreal biomass data. The rubber -cocoa intercropping carbon stock was $106.91 \mathrm{tC} / \mathrm{ha}$, which corresponded to $393 \mathrm{CERs} / \mathrm{ha}$. The results lead to the following conclusion: the arboreal biomass carbon stock, particularly of the rubber trees, qualify the rubber-cocoa intercropping as a CERs generation promising activity, making the intercropping an option of Clean Development Mechanism (CDM) project, with potential for approval by the United Nations Framework Convention on Climatic Changes (UNFCCC).
\end{abstract}

Keywords: Hevea brasiliensis, Theobroma cacao, carbon project and economical viability.

\footnotetext{
${ }^{1}$ Recebido em 04.11.05 e aceito para publicação em 13.09.2006.

${ }^{2}$ Programa de Pós-Graduação em Ciência Florestal da Universidade Federal de Viçosa. E-mail: <mkcotta@ yahoo.com.br>.

${ }^{3}$ Departamento de Engenharia Florestal da Universidade Federal de Viçosa, 36570-000 Viçosa-MG. E-mail: <jacovine@ufv.br>.

${ }^{4}$ Comissão Executiva do Plano da Lavoura Cacaueira - CEPLAC. Cx.P. 07, 45600-970 Itabuna-BA.
} 


\section{INTRODUÇÃO}

A grande quantidade de gases emitidos decorrentes das atividades humanas, principalmente da queima de combustíveis fósseis e do desmatamento, vem ocasionando acúmulo de carbono em forma de $\mathrm{CO}_{2}$ na atmosfera e, conseqüentemente, a elevação da temperatura global.

Buscando estabelecer medidas para diminuição da emissão dos Gases de Efeito-Estufa (GEE), foi ratificado o Protocolo de Kyoto, que determina prazos e metas para reduções dos GEE e prevê a utilização de mecanismos de flexibilização. Dentre esses mecanismos, destaca-se o Mecanismo de Desenvolvimento Limpo (MDL), o qual permite aos países desenvolvidos compensarem suas emissões por meio de financiamentos de projetos ambientais sediados em países em desenvolvimento.

A atividade florestal surge como uma alternativa de projeto de MDL, visto que as florestas, além de servirem como fonte renovável de energia, acumulam carbono em sua biomassa. Esse tipo de projeto é conhecido como projeto de "seqüestro" de carbono. Segundo Nogueira e Trossero (2004), o "seqüestro" de carbono pelas florestas é considerado uma alternativa capaz de mitigar o aumento dos GEE, permitindo uma solução temporária e efetiva. Uma vez comprovada a redução de emissão dos GEE, o país "hospedeiro" do projeto pode auferir certificados que comprovem a dita redução. Esses certificados são denominados Certificados de Emissões Reduzidas (CERs).

Nesse contexto, o consórcio seringueira-cacau apresenta-se como uma opção de projeto para geração de CERs e candidato à elegibilidade como projeto de MDL. A seringueira normalmente tem sido plantada em amplos espaçamentos, permitindo a consorciação com outras culturas. O cacau é bastante indicado para esse fim, visto ser uma cultura que necessita de sombreamento desde a fase de implantação até a fase produtiva.

Para Albrecht e Kandji (2003), o "seqüestro" de carbono por culturas perenes apresenta vantagens comparativas, pois, além de estocar o carbono por longo período de tempo, a exploração econômica dessas culturas não necessariamente termina com o corte da madeira, o que provavelmente levaria à emissão de $\mathrm{CO}_{2}$ para a atmosfera.
Considerando a possibilidade de o consórcio seringueira-cacau gerar CERs e se tornar atividade de projeto elegível no MDL, foi elaborado este trabalho. Além disso, constatou-se que ainda existem muitas controvérsias e indefinições quanto à aprovação de projetos florestais para a geração de créditos de carbono, o que leva à necessidade de desenvolvimento de pesquisas sobre o assunto. Desse modo, o objetivo deste estudo foi analisar a viabilidade econômica do consórcio da seringueira aos 34 anos com o cacau aos 30 anos de idade com e sem a inclusão dos créditos de carbono.

\section{MATERIAL E MÉTODOS}

\subsection{Quantificação da biomassa, estimativa de carbono e contabilização dos CERs}

Os dados de biomassa e de estoque de carbono utilizados para se realizar a análise econômica foram adaptados do estudo realizado por Cotta (2005) (Quadro 1). Nesse estudo, a quantificação da biomassa arbórea foi realizada em uma área de 2,2 ha das Fazendas Reunidas Vale do Juliana, Bahia, onde haviam consorciadas seringueiras do clone FX 2261 com 34 anos de idade e cacaueiros enxertados, com 6 anos de idade.

A estimativa do estoque de carbono nas árvores foi obtida considerando-se que o porcentual de carbono na biomassa seca seja 50\%, valor sugerido por Dewar e Cannel (1992) e Soares e Oliveira (2002).

Para verificar a viabilidade do projeto, a quantificação dos CERs e a análise econômica foram realizadas para uma simulação do consórcio da seringueira aos 34 anos com o cacaueiro aos 30 anos de idade. Para isso, considerou-se um horizonte de planejamento de 34 anos para o projeto, o plantio da seringueira no primeiro ano e o do cacaueiro no quarto ano.

A estimativa do estoque de carbono da seringueira até aos 30 anos e do cacaueiro até o sexto ano foi realizada com base nos levantamentos de campo de Cotta (2005). A estimativa da estocagem de carbono do sétimo ao trigésimo ano do cacaueiro foi feita com base no trabalho de Teixeira e Oliveira (1999), que encontraram incremento médio de 0,71 tC/ha.ano, para cacaueiro com idade de 20 anos.

A conversão do carbono em $\mathrm{CO}_{2}$ equivalente $\left(\mathrm{CO}_{2 \mathrm{eq}}\right)$ foi realizada multiplicando-se o total de carbono estocado por 44/12 (razão do peso molecular do $\mathrm{CO}_{2}$ e do carbono), 
ou seja, uma tonelada de carbono corresponde a 3,67 toneladas de $\mathrm{CO}_{2}$ eq.. Assim, os CERs gerados pelo consórcio corresponderão a $393 \mathrm{tCO}_{2}$ eq./ha, em que 311 tCO2eq./ha são referentes à seringueira e 82 tCO $\mathrm{tCq}_{2}$ eq ha ao cacaueiro, ou seja, as $393 \mathrm{tCO}_{2}$ eq./ha corresponderão a 393 CERs/ha.

\subsection{Fluxo de caixa e taxa de desconto utilizada}

Os custos e as receitas incidentes no projeto foram ordenados anualmente em um fluxo de caixa, segundo Rezende e Oliveira (2001).

A taxa de desconto aplicada foi de $10 \%$ ao ano, a qual é recomendada pelo Centro de Estudos Integrados sobre o Meio Ambiente e Mudanças Climáticas do Ministério do Meio Ambiente, no documento que trata dos critérios de elegibilidade e indicadores de sustentabilidade para a avaliação de projetos candidatos ao MDL (BRASIL, 2002).

\subsection{Composição dos custos}

Os custos utilizados para se proceder à análise econômica foram aqueles relacionados à mão-de-obra e aos insumos usados para a realização das seguintes atividades: implantação do consórcio (preparo da área, aquisição de mudas e plantio), manutenção (tratos culturais), e produção (sangria e colheita/beneficiamento do cacau) (Quadro 2). O custo da terra foi de R \$1.100,00/ ha, valor referente a terras com pastagens de baixo suporte no sudeste da Bahia (AGRIANUAL, 2004). Considerou-se para cada ano de produção um valor de $10 \%$ do valor da terra, por hectare.

As informações sobre os coeficientes técnicos e as atividades realizadas no consórcio tiveram como referência a Comissão Executiva do Plano da Lavoura Cacaueira - CEPLAC. Informações complementares foram obtidas em Gramacho et al. (1992), Virgens Filho (2002) e AgrianuaL (2004).

Na determinação dos custos de mão-de-obra para a realização das atividades, levou-se em consideração o valor do salário mínimo de $\mathrm{R} \$ 260,00$ com acréscimo de $50 \%$, referente aos encargos sociais, valor médio adaptado daquele sugerido pelo Sebrae (2002), e 22 dias úteis trabalhados no mês. Os preços dos insumos foram obtidos através de pesquisas de mercado e consultas ao Agrianual (2004).

O custo referente ao projeto de carbono para a obtenção dos CERs refere-se às seguintes etapas: definição e elaboração do projeto, estudo de viabilidade, negociação, determinação da linha de base, plano de monitoramento, certificação, e validação.

Quadro 1 - Estoque médio e porcentual de carbono nos compartimentos da seringueira com 34 anos de idade e do cacaueiro com 6 anos de idade, por hectare

Table 1 - Average carbon stock and percentage in the compartments of 34-year-old rubber tree and 6-year-old cacao tree per hectare

\begin{tabular}{|c|c|c|c|c|}
\hline \multirow{2}{*}{$\begin{array}{l}\text { Compartimento } \\
\text { da árvore }\end{array}$} & \multicolumn{2}{|c|}{ Seringueira } & \multicolumn{2}{|c|}{ Cacau } \\
\hline & Carbono (tC/ha) & Valor Percentual (\%) & Carbono (tC/ha) & Valor Percentual (\%) \\
\hline \multicolumn{5}{|l|}{ Parte Aérea } \\
\hline Folhas & 1,85 & 2,00 & 1,02 & 20,00 \\
\hline Tronco & 19,22 & 23,00 & 0,62 & 12,00 \\
\hline \multicolumn{5}{|l|}{ Galhos } \\
\hline Grossos & 22,89 & 27,00 & 1,46 & 28,00 \\
\hline Médios & 11,15 & 13,00 & 0,39 & 7,00 \\
\hline Finos & 13,30 & 16,00 & 0,28 & 5,00 \\
\hline Subtotal & 68,41 & 81,00 & 3,77 & 72,00 \\
\hline \multicolumn{5}{|l|}{ Raízes } \\
\hline Pivotante & 11,09 & 13,00 & 0,62 & 12,00 \\
\hline \multicolumn{5}{|l|}{ Laterais } \\
\hline Grossas & 2,25 & 3,00 & 0,24 & 5,00 \\
\hline Médias & 2,04 & 2,00 & 0,27 & 5,00 \\
\hline Finas & 0,86 & 1,00 & 0,32 & 6,00 \\
\hline Subtotal & 16,24 & 19,00 & 1,45 & 28,00 \\
\hline Peso total & 84,65 & 100,00 & 5,22 & 100,00 \\
\hline
\end{tabular}

Fonte: Cotta, 2005 
Quadro 2 - Custos anuais das atividades de implantação, manutenção e produção do consórcio seringueira-cacau Table 2 - Annual costs of the activities of implantation, management and production of the rubber-cacao intercropping

\begin{tabular}{|c|c|c|c|c|c|c|}
\hline \multirow[b]{3}{*}{ Ano } & \multicolumn{6}{|c|}{ Custos do Consórcio (R \$/ha)/Atividades } \\
\hline & \multicolumn{3}{|c|}{ Implantação } & \multirow{2}{*}{$\begin{array}{c}\text { Manutenção } \\
\text { Tratos culturais }\end{array}$} & \multicolumn{2}{|c|}{ Produção } \\
\hline & Preparo da área & Mudas & Plantio & & Sangria & $\begin{array}{c}\text { Colheita/beneficiamento } \\
\text { do cacau }\end{array}$ \\
\hline 0 & 913,56 & $1.050,00$ & 897,30 & - & - & - \\
\hline 1 & - & - & - & $1.351,00$ & - & - \\
\hline 2 & - & - & - & $1.042,68$ & - & - \\
\hline 3 & - & - & - & $1.132,68$ & - & - \\
\hline 4 & 118,88 & 612,50 & $1.257,19$ & 985,76 & - & - \\
\hline 5 & - & - & - & $1.129,34$ & - & - \\
\hline 6 & - & - & - & $1.546,01$ & - & - \\
\hline 7 & - & - & - & $1.532,22$ & $1.460,00$ & - \\
\hline 8 & - & - & - & $1.593,06$ & $1.162,00$ & 35,44 \\
\hline 9 & - & - & - & $1.807,94$ & $1.172,00$ & 177,20 \\
\hline 10 & - & - & - & $1.678,48$ & $1.162,00$ & 212,64 \\
\hline 11 & - & - & - & $1.678,48$ & $1.172,00$ & 212,64 \\
\hline 12 & - & - & - & $1.893,36$ & $1.450,00$ & 212,64 \\
\hline 13 & - & - & - & $1.678,48$ & $1.172,00$ & 318,96 \\
\hline 14 & - & - & - & $1.678,48$ & $1.162,00$ & 761,96 \\
\hline 15 & - & - & - & $1.893,36$ & $1.172,00$ & 761,96 \\
\hline 16 & - & - & - & $1.678,48$ & $1.162,00$ & 761,96 \\
\hline 17 & - & - & - & $1.678,48$ & $1.460,00$ & 761,96 \\
\hline 18 & - & - & - & $1.893,36$ & $1.162,00$ & 761,96 \\
\hline 19 & - & - & - & $1.678,48$ & $1.172,00$ & 761,96 \\
\hline 20 & - & - & - & $1.678,48$ & $1.162,00$ & 761,96 \\
\hline 21 & - & - & - & $1.893,36$ & $1.172,00$ & 761,96 \\
\hline 22 & - & - & - & $1.678,48$ & $1.450,00$ & 761,96 \\
\hline 23 & - & - & - & $1.678,48$ & $1.172,00$ & 761,96 \\
\hline 24 & - & - & - & $1.893,36$ & $1.162,00$ & 761,96 \\
\hline 25 & - & - & - & $1.678,48$ & $1.172,00$ & 761,96 \\
\hline 26 & - & - & - & $1.678,48$ & $1.162,00$ & 761,96 \\
\hline 27 & - & - & - & $1.893,36$ & $1.460,00$ & 761,96 \\
\hline 28 & - & - & - & $1.678,48$ & $1.162,00$ & 761,96 \\
\hline 29 & - & - & - & $1.678,48$ & $1.172,00$ & 761,96 \\
\hline 30 & - & - & - & $1.893,36$ & $1.162,00$ & 761,96 \\
\hline 31 & - & - & - & $1.678,48$ & $1.172,00$ & 761,96 \\
\hline 32 & - & - & - & $1.678,48$ & $1.450,00$ & 761,96 \\
\hline 33 & - & - & - & $1.893,36$ & $1.172,00$ & 761,96 \\
\hline 34 & - & - & - & $1.678,48$ & $1.162,00$ & 761,96 \\
\hline Total & $1.032,44$ & $1.662,50$ & $2.154,49$ & $55.801,82$ & $34.404,00$ & $17.170,68$ \\
\hline
\end{tabular}

Fonte: Virgens Filho (2002).

No presente estudo, o custo para obtenção dos CERs foi estimado considerando-se um custo fixo por projeto de US $\$ 260,000(\mathrm{R} \$ 728.000,00$, considerando US $\$ 1.00$ igual a R\$2,80), valor sugerido pelo Banco Mundial (2004) e um tamanho de projeto que resulte na absorção de 50.000 tCO $\mathrm{C}_{2}$ eq./ano, o qual, segundo Haites (2004), é o tamanho mínimo de projeto economicamente viável para geração de CERs. Com base nesse tamanho mínimo de projeto e na capacidade de geração de CERs do consórcio seringueira-cacau, que foi de 11,55 CERs/ha.ano, devido ao acúmulo de $393 \mathrm{tCO}_{2}$ eq./ha no final dos 34 anos do projeto, estimou-se que será necessária uma área de 4.329 $\mathrm{ha}\left(50.000 \mathrm{tCO}_{2 \mathrm{eq}}\right.$ /ano divididas por 11,55 $\mathrm{tCO}_{2}$ eq./ha.ano) para a absorção de $50.000 \mathrm{tCO}_{2}$ eq./ano, o que equivale a 50.000 CERs/ano. Assim, o custo para elaboração do projeto para obtenção dos CERs, por hectare, foi estimado diluindo-se o custo fixo do projeto pela área de plantio, correspondendo ao valor de $\mathrm{R} \$ 168,16 / \mathrm{ha}(\mathrm{R} \$ 728.000,00$ divididos por 4.329 ha). Considerou-se que esse custo ocorreu no ano de implantação do projeto florestal.

R. Árvore, Viçosa-MG, v.30, n.6, p.969-979, 2006 


\subsection{Produção do consórcio e composição das receitas}

A produção anual de amêndoas de cacau, em arrobas por hectare, e a produção de látex, em quilo de borracha seca por hectare (Quadro 3), tiveram como referência o estudo de Virgens Filho (2002). O preço considerado para venda da borracha seca foi de $\mathrm{R} \$ 2.670,00$ a tonelada (BORRACHA NATURAL BRASILEIRA, 2004) e o do cacau, de R \$70,00 a arroba (INTERNATIONALCOCOA
ORGANIZATION, 2004). O preço da tonelada de $\mathrm{CO}_{2}$ eq. foi de US\$ 5.50, conforme sugerido em estudos realizados por Haites (2004) e Lecocq (2004). Considerou-se que a receita obtida com os CERs ocorreu no ano 1 do projeto.

\subsection{Análise Econômica}

A análise econômica foi realizada utilizando-se os critérios a seguir, de acordo com Rezende e Oliveira (2000) e Silva et al. (2002).

Quadro 3-Custo, receita e produção anual do consórcio seringueira-cacau sem os CERs

Table 3 - Cost, revenues and annual production the rubber - cacao intercropping without the CERs

\begin{tabular}{|c|c|c|c|c|c|}
\hline \multirow[b]{2}{*}{ Ano } & \multirow[b]{2}{*}{ Atividades } & \multirow[b]{2}{*}{$\begin{array}{l}\text { Custo } \\
(\mathrm{R} \$ / \mathrm{ha})\end{array}$} & \multicolumn{2}{|c|}{ Produção } & \multirow[b]{2}{*}{$\begin{array}{l}\text { Receita } \\
\text { (R\$/ha) }\end{array}$} \\
\hline & & & $\begin{array}{l}\text { Seringueira } \\
(\mathrm{kg} . \mathrm{BS} * / \mathrm{ha})\end{array}$ & $\begin{array}{l}\text { Cacau } \\
\text { (@/ha) }\end{array}$ & \\
\hline 0 & Implantação Seringueira & $2.860,86$ & - & - & - \\
\hline 1 & Manutenção & $1.351,00$ & - & - & - \\
\hline 2 & Manutenção & $1.042,68$ & - & - & - \\
\hline 3 & Manutenção & $1.132,68$ & - & - & - \\
\hline 4 & Manut./Implant. Cacau & $2.974,33$ & - & - & - \\
\hline 5 & Manutenção & $1.129,34$ & - & - & - \\
\hline 6 & Manutenção & $1.546,10$ & - & - & - \\
\hline 7 & Manutenção/Produção & $2.992,22$ & 338,00 & - & 902,46 \\
\hline 8 & Manutenção/Produção & $2.790,50$ & 663,00 & 18,00 & $3.030,21$ \\
\hline 9 & Manutenção/Produção & $3.157,14$ & $1.025,00$ & 36,00 & $5.256,75$ \\
\hline 10 & Manutenção/Produção & $3.053,12$ & $1.266,00$ & 60,00 & $7.580,22$ \\
\hline 11 & Manutenção/Produção & $3.063,12$ & $1.447,00$ & 60,00 & $8.063,49$ \\
\hline 12 & Manutenção/Produção & $3.556,00$ & $1.447,00$ & 60,00 & $8.063,49$ \\
\hline 13 & Manutenção/Produção & $3.169,44$ & $1.447,00$ & 60,00 & $8.063,49$ \\
\hline 14 & Manutenção/Produção & $3.602,44$ & $1.447,00$ & 60,00 & $8.063,49$ \\
\hline 15 & Manutenção/Produção & $3.827,32$ & $1.447,00$ & 60,00 & $8.063,49$ \\
\hline 16 & Manutenção/Produção & $3.602,44$ & $1.447,00$ & 60,00 & $8.063,49$ \\
\hline 17 & Manutenção/Produção & $3.900,44$ & $1.447,00$ & 60,00 & $8.063,49$ \\
\hline 18 & Manutenção/Produção & $3.817,32$ & $1.447,00$ & 60,00 & $8.063,49$ \\
\hline 19 & Manutenção/Produção & $3.612,44$ & $1.447,00$ & 60,00 & $8.063,49$ \\
\hline 20 & Manutenção/Produção & $3.602,44$ & $1.447,00$ & 60,00 & $8.063,49$ \\
\hline 21 & Manutenção/Produção & $3.827,32$ & $1.447,00$ & 60,00 & $8.063,49$ \\
\hline 22 & Manutenção/Produção & $3.890,44$ & $1.447,00$ & 60,00 & $8.063,49$ \\
\hline 23 & Manutenção/Produção & $3.612,44$ & $1.447,00$ & 60,00 & $8.063,49$ \\
\hline 24 & Manutenção/Produção & $3.817,32$ & $1.447,00$ & 60,00 & $8.063,49$ \\
\hline 25 & Manutenção/Produção & $3.612,44$ & $1.447,00$ & 60,00 & $8.063,49$ \\
\hline 26 & Manutenção/Produção & $3.602,44$ & $1.447,00$ & 60,00 & $8.063,49$ \\
\hline 27 & Manutenção/Produção & $4.115,32$ & $1.447,00$ & 60,00 & $8.063,49$ \\
\hline 28 & Manutenção/Produção & $3.602,44$ & $1.447,00$ & 60,00 & $8.063,49$ \\
\hline 29 & Manutenção/Produção & $3.612,44$ & $1.447,00$ & 60,00 & $8.063,49$ \\
\hline 30 & Manutenção/Produção & $3.817,32$ & $1.447,00$ & 60,00 & $8.063,49$ \\
\hline 31 & Manutenção/Produção & $3.612,44$ & $1.302,00$ & 60,00 & $7.676,34$ \\
\hline 32 & Manutenção/Produção & $3.890,44$ & $1.107,00$ & 60,00 & $7.155,69$ \\
\hline 33 & Manutenção/Produção & $3.827,32$ & 886,00 & 60,00 & $6.565,62$ \\
\hline 34 & Manutenção/Produção & $3.602,44$ & 663,00 & 60,00 & $5.970,21$ \\
\hline
\end{tabular}

Fonte: Virgens Filho (2002)

* BS refere-se à Borracha Seca. 


\subsubsection{Valor Presente Líquido - VPL}

A viabilidade econômica de um projeto analisado pelo método do VPL é indicada pela diferença positiva entre as receitas e os custos, atualizados, a determinada taxa de desconto. Um projeto será economicamente viável se seu VPL for positivo a determinada taxa de juros.

$$
V P L=\sum_{j=0}^{n} R j(1+i)^{-j}-\sum_{j=0}^{n} C j(1+i)^{-j}
$$

em que:

$\mathrm{VPL}=$ valor presente líquido $\mathrm{R}_{\mathrm{j}}=$ receita no ano $\mathrm{j} ; \mathrm{C}_{\mathrm{j}}=$ custo no ano $\mathrm{j} ; \mathrm{i}=$ taxa de desconto $\mathrm{j}=$ período de ocorrência do custo ou da receita; e $n=$ duração do projeto, em anos.

\subsubsection{Taxa Interna de Retorno - TIR}

A TIR representa a taxa de desconto que iguala o valor presente das receitas ao valor presente dos custos. Um projeto será economicamente viável se sua TIR for maior do que uma taxa de desconto alternativa do capital, denominada taxa mínima de atratividade.

$$
\sum_{j=0}^{n} R j(1+i)^{-j}=\sum_{j=0}^{n} C j(1+i)^{-j}
$$

em que:

$\mathrm{R}_{\mathrm{j}}=$ receita no ano $\mathrm{j} ; \mathrm{C}_{\mathrm{j}}=$ custo no ano $\mathrm{j} ; \mathrm{i}=$ taxa de desconto; $\mathrm{j}=$ período de ocorrência do custo ou da receita; e $\mathrm{n}=$ duração do projeto, em anos.

\subsubsection{Valor Anual Equivalente - VAE}

O VAE transforma o valor atual do projeto em fluxos de receitas ou custos periódicos contínuos, equivalentes ao valor atual, durante a vida útil do projeto. Um projeto será economicamente viável se apresentar VAE positivo.

$$
V A E=\frac{V P L^{*} i}{1-(1+i)^{-n}}
$$

em que:

$\mathrm{VAE}=$ valor anual equivalente; $\mathrm{VPL}=$ valor presente líquido; $\mathrm{i}=$ taxa de desconto; e $\mathrm{n}=$ duração do projeto, em anos.

R. Árvore, Viçosa-MG, v.30, n.6, p.969-979, 2006

\subsection{Análise de sensibilidade}

A análise de sensibilidade foi realizada para o critério VPL, considerando-se o cenário onde foi incluída a venda dos CERs. Para tanto, a taxa de juros, o item de custo da mão-de-obra e os preços da borracha, do cacau e dos CERs foram submetidos a uma variação de seus valores de $-20 \%$ e $+20 \%$. Utilizando a ferramenta "atingir meta" do programa Microsoft Excel, foi analisado quanto o valor do salário mínimo e os preços da borracha, do cacau e dos CERs podem variar para que o projeto se mantenha viável, quanto ao critério VPL.

\section{RESULTADOS E DISCUSSÃO}

\subsection{Análise econômica}

\subsubsection{Fluxo de caixa do consórcio sem os CERs}

As variações no fluxo de caixa do consórcio seringueira-cacau no decorrer do ciclo de produção foram atribuídas às alterações dos custos e das receitas. As variações dos custos foram influenciadas principalmente pelas manutenções anuais e pelos custos referentes à sangria e colheita/beneficiamento do cacau. Já as alterações das receitas foram decorrentes das variações na produção de látex e de cacau no decorrer dos anos (Quadro 3).

O fluxo de caixa corrente do consórcio seringueiracacau sem a inclusão dos CERs apresentou-se negativo até o $7^{\circ}$ ano, quando se iniciou a extração do látex, passando a ser positivo a partir do $8^{\circ}$ ano, com a inclusão das receitas do cacau (Quadro 4). O saldo negativo nos primeiros anos é justificado pelo fato de o consórcio não apresentar receitas até o $6^{\circ}$ ano e pelo incremento dos custos com a implantação do cacau e a aquisição de materiais para o início da extração do látex.

O fluxo de caixa acumulado indicou que para o cenário sem os CERs o lucro do projeto passou a ocorrer somente a partir do $16^{\circ}$ ano, embora a partir do $8^{\circ}$ ano as receitas tenham sido superiores aos custos, mantendose, assim, até o final do ciclo do projeto (Quadro 4).

O comportamento observado no fluxo de caixa desse projeto não difere daqueles geralmente encontrados na maioria dos projetos florestais. Altos custos iniciais e receitas no longo prazo são características que, muitas vezes, tornam os projetos dessa natureza pouco atrativos. 
Quadro 4 - Custo, receita e fluxo de caixa anual (corrente, atualizado e acumulado) para o consórcio seringueira-cacau sem a inclusão dos CERs

Table 4-Cost, revenues and cash flow (current, actual and accumulated) of rubber - cacao intercropping without CERs

\begin{tabular}{|c|c|c|c|c|c|}
\hline \multirow[t]{2}{*}{ Ano } & \multirow[t]{2}{*}{ Custo Total $\mathrm{R} \$ / \mathrm{ha}$} & \multirow[t]{2}{*}{ Receita $\mathrm{R} \$ / \mathrm{ha}$} & \multicolumn{3}{|c|}{ Fluxo de Caixa $\mathrm{R} \$ / \mathrm{ha}$} \\
\hline & & & Corrente & Atualizado & Acumulado \\
\hline 0 & $2.860,86$ & - & $-2.860,86$ & $-2.860,86$ & $-2.860,86$ \\
\hline 1 & $1.351,00$ & - & $-1.351,00$ & $-1.228,18$ & $-4.089,04$ \\
\hline 2 & $1.042,68$ & - & $-1.042,68$ & $-861,72$ & $-4.950,76$ \\
\hline 3 & $1.132,68$ & - & $-1.132,68$ & $-851,00$ & $-5.801,76$ \\
\hline 4 & $2.974,33$ & - & $-2.974,33$ & $-2.031,51$ & $-7.833,27$ \\
\hline 5 & $1.129,34$ & - & $-1.129,34$ & $-701,23$ & $-8.534,50$ \\
\hline 6 & $1.546,10$ & - & $-1.546,10$ & $-872,73$ & $-9.407,23$ \\
\hline 7 & $2.992,22$ & 902,46 & $-2.089,76$ & $-1.072,38$ & $-10.479,61$ \\
\hline 8 & $2.790,50$ & $3.030,21$ & 239,71 & 111,83 & $-10.367,78$ \\
\hline 9 & $3.157,14$ & $5.256,75$ & $2.099,61$ & 890,44 & $-9.477,34$ \\
\hline 10 & $3.053,12$ & $7.580,22$ & $4.527,10$ & $1.745,39$ & $-7.731,95$ \\
\hline 11 & $3.063,12$ & $8.063,49$ & $5.000,37$ & $1.752,60$ & $-5.979,35$ \\
\hline 12 & $3.556,00$ & $8.063,49$ & $4.507,49$ & $1.436,23$ & $-4.543,13$ \\
\hline 13 & $3.169,44$ & $8.063,49$ & $4.894,05$ & $1.417,63$ & $-3.125,49$ \\
\hline 14 & $3.602,44$ & $8.063,49$ & $4.461,05$ & $1.174,73$ & $-1.950,76$ \\
\hline 15 & $3.827,32$ & $8.063,49$ & $4.236,17$ & $1.014,11$ & $-936,65$ \\
\hline 16 & $3.602,44$ & $8.063,49$ & $4.461,05$ & 970,85 & 34,20 \\
\hline 17 & $3.900,44$ & $8.063,49$ & $4.163,05$ & 823,64 & 857,84 \\
\hline 18 & $3.817,32$ & $8.063,49$ & $4.246,17$ & 763,71 & $1.621,55$ \\
\hline 19 & $3.612,44$ & $8.063,49$ & $4.451,05$ & 727,78 & $2.349,33$ \\
\hline 20 & $3.602,44$ & $8.063,49$ & $4.461,05$ & 663,11 & $3.012,44$ \\
\hline 21 & $3.827,32$ & $8.063,49$ & $4.236,17$ & 572,44 & $3.584,87$ \\
\hline 22 & $3.890,44$ & $8.063,49$ & $4.173,05$ & 512,64 & $4.097,52$ \\
\hline 23 & $3.612,44$ & $8.063,49$ & $4.451,05$ & 497,09 & $4.594,60$ \\
\hline 24 & $3.817,32$ & $8.063,49$ & $4.246,17$ & 431,09 & $5.025,70$ \\
\hline 25 & $3.612,44$ & $8.063,49$ & $4.451,05$ & 410,81 & $5.436,51$ \\
\hline 26 & $3.602,44$ & $8.063,49$ & $4.461,05$ & 374,31 & $5.810,82$ \\
\hline 27 & $4.115,32$ & $8.063,49$ & $3.948,17$ & 301,16 & $6.111,97$ \\
\hline 28 & $3.602,44$ & $8.063,49$ & $4.461,05$ & 309,34 & $6.421,32$ \\
\hline 29 & $3.612,44$ & $8.063,49$ & $4.451,05$ & 280,59 & $6.701,91$ \\
\hline 30 & $3.817,32$ & $8.063,49$ & $4.246,17$ & 243,34 & $6.945,25$ \\
\hline 31 & $3.612,44$ & $7.676,34$ & $4.063,90$ & 211,72 & $7.156,97$ \\
\hline 32 & $3.890,44$ & $7.155,69$ & $3.265,25$ & 154,65 & $7.311,63$ \\
\hline 33 & $3.827,32$ & $6.565,62$ & $2.738,30$ & 117,90 & $7.429,53$ \\
\hline 34 & $3.602,44$ & $5.970,21$ & $2.367,77$ & 92,68 & $7.522,21$ \\
\hline
\end{tabular}

\subsubsection{Fluxo de caixa do consórcio com os CERs}

O fluxo de caixa do cenário considerando a venda dos créditos de carbono diferenciou-se do anterior devido à inclusão do custo do projeto para geração dos CERs (R \$168,16/ha ), no ano de implantação florestal, e ao incremento na receita ( $\mathrm{R} \$ 6.052,20 / \mathrm{ha})$, provenientes da venda desses créditos (Quadro 5).

As receita dos CERs no ano 1 permitiu ao investidor cobrir os custos de implantação da seringueira e possibilitou antecipar o retorno do investimento do $16^{\circ}$ ano para o $12^{\circ}$ ano, em comparação com o cenário anterior. $\mathrm{O}$ fluxo de caixa acumulado nos anos seguintes comprovou que a inclusão dos créditos de carbono ao consórcio é uma opção para tornar a atividade mais rentável.

Pesquisas de mercado realizadas por Haites (2004) apontaram o valor atual para as unidades de redução na emissão de aproximadamente US\$5.50, podendo alcançar o preço de US\$11.00 em 2010. Em uma sondagem de preços realizada em outubro de 2003, no encontro da International Emissions Trading Association, indicouse que o preço médio para a tonelada de $\mathrm{CO}_{2}$ será de US\$14.30 no ano de 2010. Outra estimativa de preço para $\mathrm{O} \mathrm{CO}_{2}$ em 2010 foi feita pela Point Carbon, que sugeriu um valor de US\$9.90 a tonelada de $\mathrm{CO}_{2}$.

R. Árvore, Viçosa-MG, v.30, n.6, p.969-979, 2006 
Quadro 5 - Custo, receita e fluxo de caixa anual (corrente, atualizado e acumulado) para o consórcio seringueira-cacau com a inclusão dos CERs

Table 5 - Cost, revenues and cash flow (current, actual and accumulated) of the rubber - cacao intercropping with CERs

\begin{tabular}{|c|c|c|c|c|c|}
\hline \multirow[t]{2}{*}{ Ano } & \multirow[t]{2}{*}{ Custo Total $\mathrm{R} \$ / \mathrm{ha}$} & \multirow[t]{2}{*}{ Receita R \$/ha } & \multicolumn{3}{|c|}{ Fluxo de Caixa $\mathrm{R} \$ / \mathrm{ha}$} \\
\hline & & & Corrente & Atualizado & Acumulado \\
\hline 0 & $3.029,02$ & - & $-3.029,02$ & $-3.029,02$ & $-3.029,02$ \\
\hline 1 & $1.351,00$ & $6.052,20$ & $4.701,20$ & $4.273,82$ & $1.244,80$ \\
\hline 2 & $1.042,68$ & - & $-1.042,68$ & $-861,72$ & 383,08 \\
\hline 3 & $1.132,68$ & - & $-1.132,68$ & $-851,00$ & $-467,92$ \\
\hline 4 & $2.974,33$ & - & $-2.974,33$ & $-2.031,51$ & $-2.499,43$ \\
\hline 5 & $1.129,34$ & - & $-1.129,34$ & $-701,23$ & $-3.200,66$ \\
\hline 6 & $1.546,10$ & - & $-1.546,10$ & $-872,73$ & $-4.073,39$ \\
\hline 7 & $2.992,22$ & 902,46 & $-2.089,76$ & $-1.072,38$ & $-5.145,77$ \\
\hline 8 & $2.790,50$ & $3.030,21$ & 239,71 & 111,83 & $-5.033,94$ \\
\hline 9 & $3.157,14$ & $5.256,75$ & $2.099,61$ & 890,44 & $-4.143,50$ \\
\hline 10 & $3.053,12$ & $7.580,22$ & $4.527,10$ & $1.745,39$ & $-2.398,11$ \\
\hline 11 & $3.063,12$ & $8.063,49$ & $5.000,37$ & $1.752,60$ & $-645,51$ \\
\hline 12 & $3.556,00$ & $8.063,49$ & $4.507,49$ & $1.436,23$ & 790,71 \\
\hline 13 & $3.169,44$ & $8.063,49$ & $4.894,05$ & $1.417,63$ & $2.208,35$ \\
\hline 14 & $3.602,44$ & $8.063,49$ & $4.461,05$ & $1.174,73$ & $3.383,08$ \\
\hline 15 & $3.827,32$ & $8.063,49$ & $4.236,17$ & $1.014,11$ & $4.397,19$ \\
\hline 16 & $3.602,44$ & $8.063,49$ & $4.461,05$ & 970,85 & $5.368,04$ \\
\hline 17 & $3.900,44$ & $8.063,49$ & $4.163,05$ & 823,64 & $6.191,68$ \\
\hline 18 & $3.817,32$ & $8.063,49$ & $4.246,17$ & 763,71 & $6.955,39$ \\
\hline 19 & $3.612,44$ & $8.063,49$ & $4.451,05$ & 727,78 & $7.683,17$ \\
\hline 20 & $3.602,44$ & $8.063,49$ & $4.461,05$ & 663,11 & $8.346,28$ \\
\hline 21 & $3.827,32$ & $8.063,49$ & $4.236,17$ & 572,44 & $8.918,71$ \\
\hline 22 & $3.890,44$ & $8.063,49$ & $4.173,05$ & 512,64 & $9.431,36$ \\
\hline 23 & $3.612,44$ & $8.063,49$ & $4.451,05$ & 497,09 & $9.928,44$ \\
\hline 24 & $3.817,32$ & $8.063,49$ & $4.246,17$ & 431,09 & $10.359,54$ \\
\hline 25 & $3.612,44$ & $8.063,49$ & $4.451,05$ & 410,81 & $10.770,35$ \\
\hline 26 & $3.602,44$ & $8.063,49$ & $4.461,05$ & 374,31 & $11.144,66$ \\
\hline 27 & $4.115,32$ & $8.063,49$ & $3.948,17$ & 301,16 & $11.445,81$ \\
\hline 28 & $3.602,44$ & $8.063,49$ & $4.461,05$ & 309,34 & $11.755,16$ \\
\hline 29 & $3.612,44$ & $8.063,49$ & $4.451,05$ & 280,59 & $12.035,75$ \\
\hline 30 & $3.817,32$ & $8.063,49$ & $4.246,17$ & 243,34 & $12.279,09$ \\
\hline 31 & $3.612,44$ & $7.676,34$ & $4.063,90$ & 211,72 & $12.490,81$ \\
\hline 32 & $3.890,44$ & $7.155,69$ & $3.265,25$ & 154,65 & $12.645,47$ \\
\hline 33 & $3.827,32$ & $6.565,62$ & $2.738,30$ & 117,90 & $12.763,37$ \\
\hline 34 & $3.602,44$ & $5.970,21$ & $2.367,77$ & 92,68 & $12.856,05$ \\
\hline
\end{tabular}

Um fator que interfere nos preços dos CERs é o tipo de projeto que vai emitir os certificados. Os CERs provenientes de projetos de florestamento e reflorestamento têm sido cotados a preços menores. Alega-se que esse tipo de projeto não oferece segurança quanto à perenidade, estando sujeito à ocorrência de sinistros (fogo, pragas), ou à eliminação para substituição por outro tipo de cultura, o que coloca em risco o atendimento aos compromissos com a redução das emissões. Fung (2000), citado por Pandey (2002), ressaltou ainda que o tempo da retenção do carbono na árvore é uma consideração importante para projetos candidatos à geração de CERs.
Com base no custo fixo do projeto dos CERs $(\mathrm{R} \$ 728.000,00)$ e no valor de venda de US\$ 5.50/tCO 2 eq. ( $\mathrm{R} \$ 15,40$, considerando US $\$ 1.00$ igual a $\mathrm{R} \$ 2,80$ ), chegouse à conclusão de que, para a receita dos CERs cobrir esse custo, seria necessária uma área mínima de 120 ha plantada com o consórcio, o que implicaria a geração de 47.160 CERs no final de 34 anos.

\subsubsection{Análise dos itens de custo}

Durante o período de 34 anos, o custo total atualizado do consórcio seringueira-cacau por hectare, sem e com os créditos de carbono, foi de $\mathrm{R} \$ 27.372,92$ e $\mathrm{R} \$ 27.541,08$, respectivamente. 
No consórcio sem os CERs, o custo atualizado, referente à manutenção, apresentou-se como o mais elevado, correspondendo a $53 \%$ do custo total, seguido pelo custo de sangria e colheita/beneficiamento do cacau $(32 \%)$ e pelo custo de implantação (15\%). Em relação ao custo total, $61 \%$ foram referentes à mãode-obra, estando distribuídos da seguinte forma: $11 \%$ na etapa de implantação, 39\% na manutenção e 50\% na sangria e colheita/beneficiamento do cacau.

Diante desses resultados, pode-se afirmar que o item mão-de-obra é o principal componente do custo total do consórcio. Esse fato é atribuído ao uso intensivo de práticas manuais em todas as etapas do cultivo da seringueira e do cacau durante quase todo o ano e, também, aos altos encargos sociais incidentes sobre a folha de pagamento dos funcionários. Por sua vez, a absorção de mão-de-obra e, conseqüentemente, a contribuição para a geração de emprego e renda no meio rural são características que fazem que o consórcio seringueira-cacau possa atender a um dos objetivos do MDL: contribuir para o desenvolvimento sustentável do país de implantação do projeto.

Para o cenário considerando os créditos de carbono, verificou-se que o custo dos CERs foi equivalente a $0,6 \%$ do custo total atualizado do projeto, por hectare. Como o custo dos CERs geralmente é fixo por projeto, pode-se inferir que esse custo por hectare pode ser diluído de acordo com o tamanho da área do projeto ou a quantidade de CERs gerados. Além disso, como a comercialização de créditos de carbono é uma atividade nova no mercado, espera-se que, à medida que novos projetos forem aprovados e as metodologias de quantificação de emissões e de reduções se tornarem mais simplificadas, esses custos sejam reduzidos, proporcionando, assim maior viabilidade aos projetos de MDL.

\subsubsection{Análise da viabilidade econômica pelos critérios VPL, TIR e VAE}

Os valores dos VPLs apontaram que o consórcio proporcionou um lucro de $\mathrm{R} \$ 7.522,21 /$ ha e $\mathrm{R} \$ 12.856,05$ / ha sem e com os créditos de carbono, respectivamente. Isso indica que a inclusão dos CERs ao projeto é uma alternativa capaz de aumentar a viabilidade do consórcio e atuar como um atrativo ao investimento em projetos dessa natureza.
Quanto à TIR, verificou-se que no cenário sem a inclusão dos CERs a TIR foi de $14,62 \%$ e com os CERs, aumentou para $24,12 \%$. A TIR de ambos os cenários é considerada razoável. Porém, as altas taxas de juros no Brasil e a propensão aos riscos que os projetos florestais apresentam são fatores que diminuem a sua atratividade, mesmo que tais projetos se apresentem viáveis.

Os valores encontrados para o VAE de $\mathrm{R} \$ 782,86 /$ ha e $\mathrm{R} \$ 1.337,98 /$ ha sem e com os CERs, respectivamente, indicaram uma boa rentabilidade, principalmente por se tratar de uma atividade que pode ser praticada por pequenos produtores rurais e que não exige o uso da mecanização, sendo os principais fatores de produção a terra e a mão-de-obra.

\subsection{Análise de sensibilidade}

Os resultados da análise de sensibilidade para o critério VPL evidenciaram que as variáveis que mais afetaram a viabilidade do consórcio foram a taxa de juros e o preço do cacau (Figura 1). O decréscimo de $20 \%$ na taxa de juros ocasionou aumento de $\mathrm{R} \$ 12.856,05$ para R\$19.222,79 no VPL, o que correspondeu a um acréscimo de $49 \%$ na viabilidade do projeto. A maior variação observada no critério VPL, devido às variações na taxa de juros, comprova que a taxa de juros é um dos principais fatores que afetam a viabilidade econômica do consórcio. Isso ocorre em razão da natureza do fluxo de caixa desse tipo de projeto, em que os custos incidem do início ao fim e as receitas só passam a ocorrer posteriormente aos custos iniciais, geralmente após o $6^{\circ}$ ano.

O acréscimo de $20 \%$ no preço dos CERs aumentou, no entanto, a viabilidade do consórcio em apenas $8,5 \%$. Isso porque a receita proveniente dos CERs representa um montante menor quando comparado com o custo total do investimento; com isso, quando incorrem os juros, a receita dos CERs é menos afetada do que os custos do projeto. Outro fator é o prazo de incidência dos juros sobre as receitas e os custos. Como os CERs são negociados no ano 1, eles sofreram menor influência da taxa de desconto.

A maior influência da variável preço do cacau no critério VPL, em relação às variáveis preço da borracha e preço dos CERs, é justificada pelo montante das receitas advindas do produto.

R. Árvore, Viçosa-MG, v.30, n.6, p.969-979, 2006 


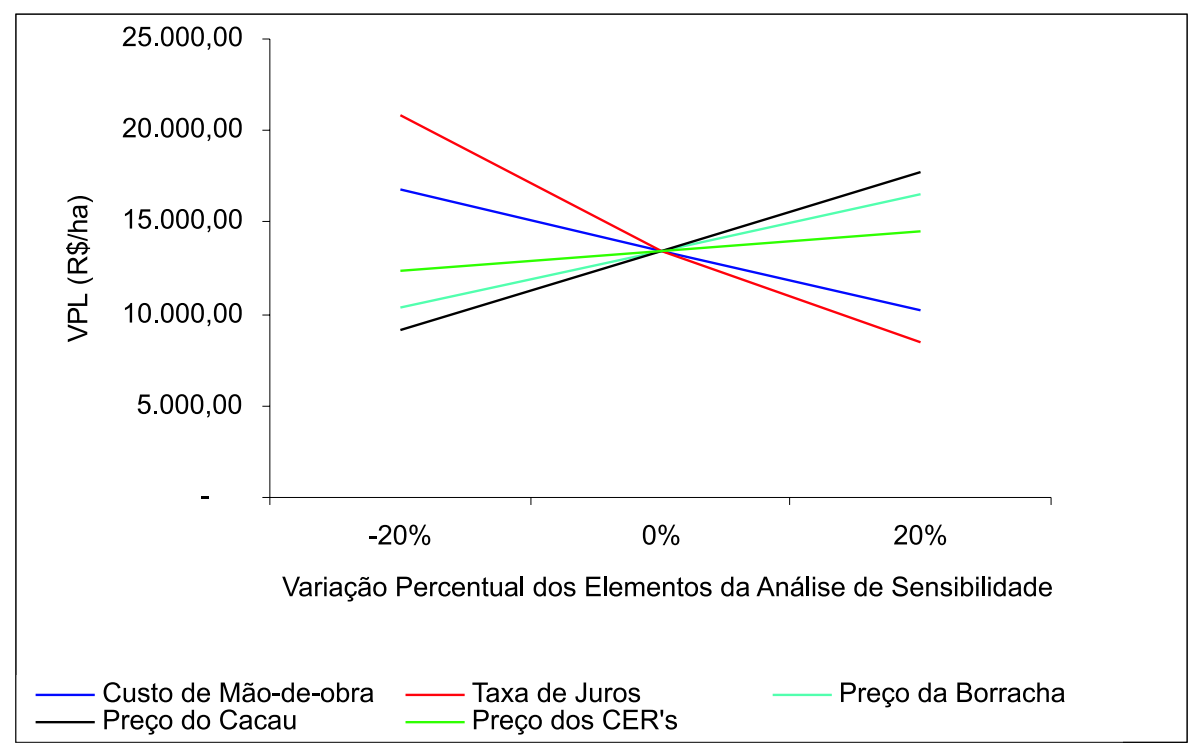

Figura 1 - Análise de sensibilidade do Valor Presente Líquido. Figure 1-Sensibility analysis of the Net Present Value.

Por meio das análises realizadas utilizando a ferramenta "atingir meta" do programa Excel, verificouse que, mantendo os custos de produção e a taxa de desconto de $10 \%$ a.a, o projeto será viável economicamente mesmo sem as receitas dos CERs.

Quando considerados os CERs, observou-se que o preço da borracha poderá sofrer redução de até $76 \%$ que o projeto continuará viável. Nessa mesma situação, constatou-se que o preço do cacau poderá cair até $72 \%$ em relação ao preço atual. Mesmo que a arroba de cacau fosse comercializada a $\mathrm{R} \$ 19,51$, o projeto manterse-ia viável.

Em relação ao item de custo mão-de-obra, que foi de $\mathrm{R} \$ 17,72 /$ dia (considerando o salário de $\mathrm{R} \$ 260,00$, encargos de $50 \%$ e 22 dias úteis por mês), observouse que esse valor poderá sofrer um acréscimo de até $78 \%$, ou seja, atingir o valor de no máximo $\mathrm{R} \$ 31,59 /$ dia que, ainda assim, o projeto manter-se ia viável.

\section{CONCLUSÕES}

Diante dos resultados apresentados neste trabalho, pode-se concluir que:

i) O estoque de carbono na biomassa arbórea, particularmente das árvores de seringueira, credencia o consórcio seringueira-cacau como uma atividade promissora na geração de CERs; ii) O custo da mãode-obra é responsável por $61 \%$ do custo total do consórcio, evidenciando-se a sua contribuição significativa na geração de emprego e renda no meio rural e, conseqüentemente, no desenvolvimento sustentável, característica desejada nos projetos de MDL; iii) O consórcio mostra-se economicamente viável com e sem os CERs, entretanto os créditos de carbono aumentam consideravelmente sua atratividade, atuando como incentivo à implementação da atividade; iv) A taxa de juros é o item de custo que mais afeta a viabilidade econômica do consórcio; v) A capacidade de estocar carbono e gerar CERs torna o consórcio seringueira-cacau uma opção de projeto de MDL, com potencial de aprovação pela Convenção Quadro das Nações Unidas sobre Mudanças Climáticas.

\section{AGRADECIMENTOS}

À Comissão Executiva do Plano da Lavoura Cacaueira-CEPLAC, pela concessão dos dados e pelo apoio ao projeto, ao Sr. Norberto Odebrecht, por disponibilizar as Fazendas Reunidas Vale do Juliana para a execução do experimento; e ao Conselho Nacional de Desenvolvimento Científico e Tecnológico (CNPq), pelo apoio financeiro. 


\section{REFERÊNCIAS BIBLIOGRÁFICAS}

AGRIANUAL 2004 - Anuário da agricultura brasileira. São Paulo: FNP Consultoria e Comércio, 2004. 456 p.

ALBRECHT, A.; KANDJI, S. T. Carbon sequestration in tropical agroforestry systems. Agriculture, Ecosystems and Environment, v. 99, n. 1, p. 15-27, 2003.

BORRACHA NATURAL BRASILEIRA. Borracha via e-mail: v.5, n. 203,23 de novembro de 2004. Disponível em: <http:// www.borrachanatural.agr.br/borrachaviaemail/ index.php>Acesso em: 19 nov. 2004.

COTTA, M. K. Quantificação de biomassa e análise econômica do consórcio seringueira-cacau para geração de créditos de carbono. 2005. 89 f. Dissertação (Mestrado em Ciência Florestal) - Universidade Federal de Viçosa, Viçosa, MG, 2005.

DEWAR, R. C.; CANNELL, M. G. R. Carbon sequestration in the trees, products and soils of forest plantations: an analysis using UK examples. Tree Physiology, v. 11, n. 1, p. 49-71, 1992.

GRAMACHO, I. C. P. et al. Cultivo e beneficiamento do cacau na Bahia. Ilhéus: CEPLAC, 1992. 124 p.

HAITES, E. Estimating the market potential for the clean development mechanism: review of models and lessons learned. Washington, DC: 2004. 102 p. Disponível em : <http//carbonfinance.org/ docs/EstimatingMarketPotential.pdf $>$ Acesso em: 10 nov. de 2004.

INTERNATIONALCOCOA ORGANIZATION - ICO. Daily Cocoa Bean Prices. Disponível em: <http://www.icco.org/prices/prices.htm> Acesso em: 16 out. 2004.

LECOCQ, F. State and trends of carbon market. Washington, DC: 2004. 42 p. Disponível em: <http//carbonfinance.org/ docs/CarbonMarketStudy2004.pdf $>$ Acesso em: 10 nov. 2004.
BRASIL. MINISTÉRIO DO MEIO AMBIENTE - MMA. Critérios de elegibilidade e indicadores de sustentabilidade para avaliação de projetos que contribuam para a mitigação das mudanças climáticas e para a promoção do desenvolvimento sustentável. Brasília: 2002. 42 p.

NOGUEIRA, L. A. H.; TROSSERO, M. A. Seqüestro de carbono por sistemas dendroenergéticos. Biomassa e Energia, v. 1, n. 2, p. 131-144, 2004.

PANDEY, D. N. Carbon sequestration in agroforestry systems. Climate Policy, v. 2, n. 4 p. 367-377, 2002.

REZENDE, J. L. P.; OLIVEIRA, A. D. Análise econômica e social de projetos florestais. Viçosa, MG: Universidade Federal de Viçosa, 2001. 389 p.

REZENDE, J. L. P.; OLIVEIRA, A. D. Problemas com o horizonte de planejamento na avaliação de projetos florestais. Revista Árvore, v. 24, n. 2, p. 127-134, 2000.

SEBRAE - MG. Plano de negócios: manual do aprendiz. Belo Horizonte: 2002. 146 p.

SILVA, M. L.; JACOVINE, L. A. G.; VALVERDE, S. R. Economia florestal. Viçosa, MG: Universidade Federal de Viçsoa, 2002. 178 p.

SOARES, C. P. B.; OLIVEIRA, M. L. R..

Equações para estimar a quantidade de carbono na parte aérea de árvores de eucalipto em Viçosa, Minas Gerais. Revista Árvore, v. 26, n. 5, p. 533-539, 2002.

TEIXEIRA, L.B.; OLIVEIRA, R. F. Biomassa vegetal e carbono orgânico em capoeiras e agroecossistemas no Nordeste do Pará. Belém: Embrapa Amazônia Oriental, 1999. 21 p. (Boletim de Pesquisa, 6).

VIRGens filho, A. C. Programa de desenvolvimento do agronegócio da borracha no Estado da Bahia PRODEAB. Ilhéus: CEPLAC, 2002. 119 p.

R. Árvore, Viçosa-MG, v.30, n.6, p.969-979, 2006 